\title{
Global Tests Evaluating the Functional Condition of Hemostasis
}

Solovyev MA ${ }^{1^{*}}$, Tutrin $I^{2}$, Udut $\mathbf{V V}^{1,2}$, Borodulina EV ${ }^{1}$ and Turenko AV ${ }^{2}$

${ }^{1}$ Research Institute of Pharmacology and Regenerative Medicine name of E.D.Goldberg, Russia

${ }^{2}$ Tomsk State University, Russia

\begin{abstract}
Control of effectiveness of global tests evaluation of the functional state regulation of blood aggregation provided an opportunity to obtain timely information about the I-II and III phases of hemostasis while using native substrate - whole blood. Ex vivo, registered during hemocoagulation of changing the viscous characteristics of whole blood, allowed to formulate the concept of fibrinogenesis permanence as in vivo existing process, defining proper hemostatic potential - integrative characteristic of the entire cycle of hemocoagulation, ensuring necessary blood fluidity and restricting extravasation of its components in case of defecting its permeability or damaging its vascular wall.
\end{abstract}

Keywords: Regulation system of aggregate blood condition; Hemocoagulation; Hemostatic potential; Whole blood; Global methods; Low-frequency thromboelastography

\section{Introduction}

In recent years, the increase of mortality rates in the world is the result of a variety of diseases associated with thrombosis or hemorrhage. Also, new data on the role of endothelial function in ensuring system RBA has been gained as well as its impact on thromboresistance, vascular tone and permeability, adhesion of blood cells and angiogenesis. This allowed considering endothelial dysfunction (ED) as a key link in the pathogenesis of atherosclerosis, coronary heart disease, hypertension cancer, diabetes, preeclampsia and many other diseases. For the analysis of system RBA, clotting, amidolytic and immunferment methods are used [1-3]. Detailed evaluation of conducted studies reveals a number of disadvantages, such as lack of standardization, continuous sample preparation, low sensitivity, and most importantly, all the methods are made on the model of citrate plasma [4-7]. Thus, constantly developing new technologies, aiming to identify the genetic thrombophilia, markers of endothelial dysfunction, antiphospholipid antibodies and etc., cannot solve the problem of timely diagnosis and prevention of thrombosis and thrombohemorrhagic complications [7-10]. The global trend - the personification of research in the mode "point-ofcare test», using "global" test evaluation system RBA, becomes popular. The main methods of this direction are the thrombin generation test (TGT), thromboelastography (TEG), turbidimetric fibrin formation (TFF), fourier-converting mechanical spectroscopy (FCMS) and lowfrequency piezoelectric thromboelastography (LFPTEG) [8,11-14]. This article uses comparative analysis in order to to determine their effectiveness in the evaluation of system RBA.

\section{Method}

The study was conducted on the basis of clinics of Research Institute of Pharmacology and Regenerative Medicine name of E.D. Goldberg involving 150 conditionally healthy volunteers (mean age 22 $\pm 6,5$ years), after signing a free and informed consent.

As a model of the medium TFF, TGT was used citrate plasma, FCMS, TEG, LFPTEG the original substance - whole blood $[8,9,14,15]$. The standardization of sample preparation stage of LFPTEG has the following conditions:

- $1 \mathrm{ml}$ of blood from the cubital vein without applying tourniquet;

- 3-component siliconized syringe, $\mathrm{V}=2,5 \mathrm{ml}$;

- $\quad$ single cuvette from medical grade plastic, $\mathrm{V}=0,45 \mathrm{ml}$;

\section{- immediately (10-12 seconds) start of research.}

We analyzed the results of "global" test evaluation system RBA such as TEG, TFF, LFPTEG and thrombin generation test (TGT). These FCMS were used as a historical control, since Russia does not have a device for carrying out this research [8]. To register the blood coagulation process using technology LFPTEG, a new technological apparatus of low-frequency piezoelectric tromboelastograph was used (hardware-software complex ARP-01M "Mednord", Russia, registration certificate Rosreestr №2010/09767) $[6,16,17]$. The analysis of coagulation graph is represented on (Figure 1).

These quantitative data were processed using the programs Microsoft Excel and SPSS 13.0. The first stage has been tested the normality of distribution of quantitative indicators using the Kolmogorov - Smirnov. Then there was a statistical description of the various groups involved in the study, using nonparametric methods. Evaluated quantitative data presented in the form of Me [LQ; UQ] (where Me - median, LQ - the bottom quartile, UQ - top quartile). To test the statistical hypotheses about the difference between the study groups used the nonparametric Mann-Whitney test (where $\mathrm{p}$ - the current level of significance) [18].

\section{Results}

During the assessment of the hemostatic potential, it is necessary to include all elements of the system RBA in the study, because one cannot exclude the effect of inescapable stressor - a standardized contact activation of coagulation process (const. V seu $\mathrm{S}$ cuvette) that is achieved by mode "point - of - care test», or studies ex vivo. Evidences show that, in order to achieve maximum reliability of obtaining the results, all studies should be performed using whole blood. Indeed, the whole blood sample, containing aliquots of endothelial producers (some of which trigger effects in a limited time interval from $1 \cdot 10^{-3}$ to $30 \mathrm{sec}$ ), formed elements of blood and plasma factors hemostasis, used for operative analysis, provides an opportunity of obtaining objective

*Corresponding author: Solovyev MA, Research Institute of Pharmacology and Regenerative Medicine name of E.D.Goldberg, 15A Nikitina street, apt.8, Tomsk oblast, Tomsk, 634029, Russian Federation, Tel: +7-913-10-888-07; E-mail: m.a.solovyev@mail.ru

Received: May 21, 2015; Accepted: June 22, 2015; Published: June 26, 2015

Citation: Solovyev MA, Tutrin II, Udut VV, Borodulina EV, Turenko AV (2015) Global Tests Evaluating the Functional Condition of Hemostasis. Clin Exp Pharmacol 5: 178. doi:10.4172/2161-1459.1000178

Copyright: (c) 2015 Solovyev MA et al. This is an open-access article distributed under the terms of the Creative Commons Attribution License, which permits unrestricted use, distribution, and reproduction in any medium, provided the original author and source are credited. 
Citation: Solovyev MA, Tutrin II, Udut VV, Borodulina EV, Turenko AV (2015) Global Tests Evaluating the Functional Condition of Hemostasis. Clin Exp Pharmacol 5: 178. doi:10.4172/2161-1459.1000178

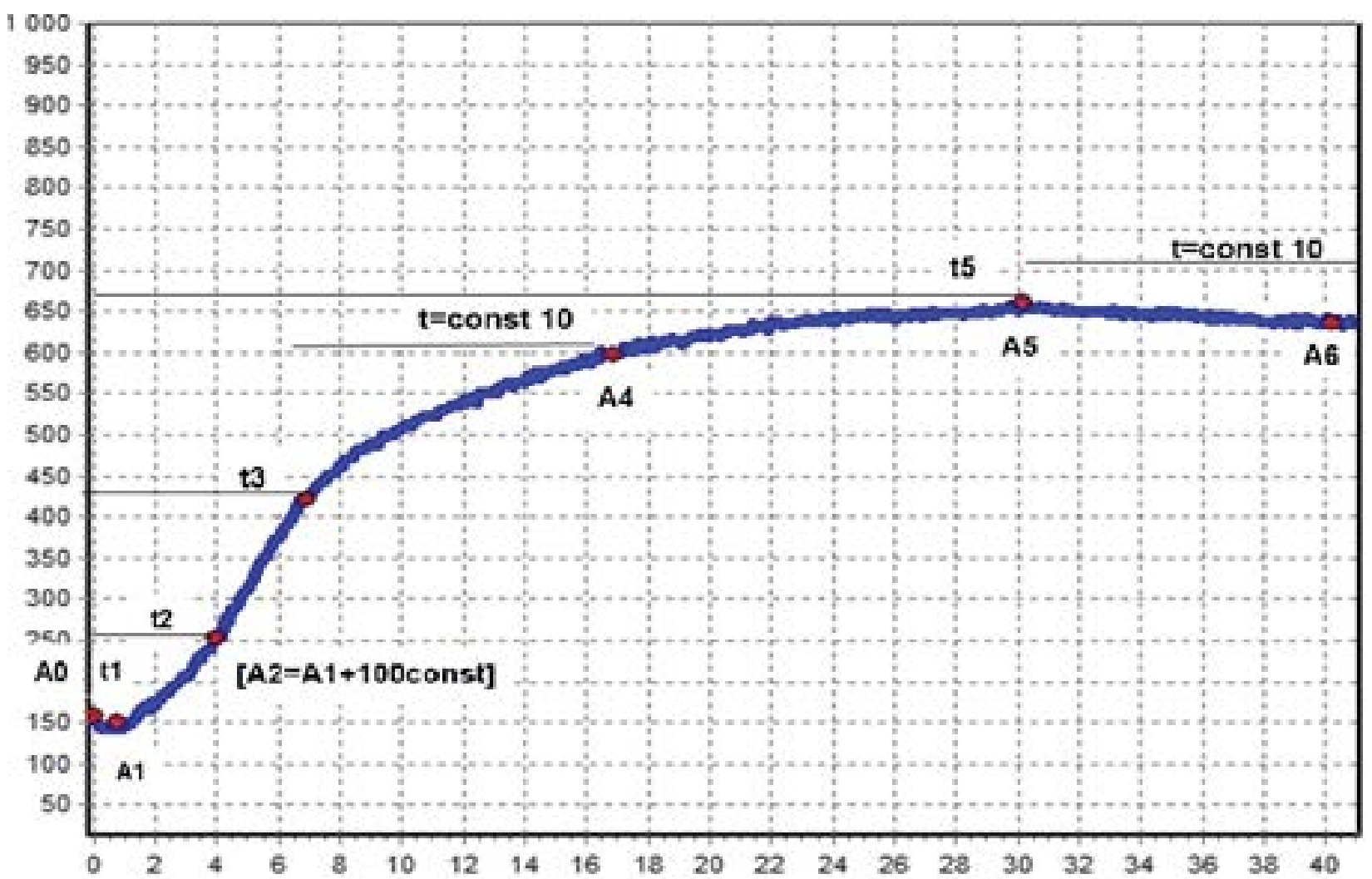

Figure 1: A graph of changes condition of blood aggregation (LFPTEG) healthy volunteer, which is estimated along the ordinate axis the amplitude research process (A1) in relative units (r.u.) and the abscissa axis - time or research (T1) in minutes A0 - initial value of the amplitude at the moment of time t0, in r.u.;

$\mathrm{t} 1$ - period of reaction (time from the beginning of research to maximum decrease of amplitude LFPTEG - A1, in minute;

A1 - maximum decrease in amplitude of the time «t 1 » (period of reaction), in r.u.;

t2 - time to achieve the amplitude A2 LFPTEG, in minute;

A2 - increase of the amplitude LFPTEG on 100 r.u. (A2-A1), in r.u.;

$\mathrm{t} 3$ - blood coagulation time (point of gelling) is determined automatically when you change tg angle of the curve $50 \%$, in minute;

A3 - amplitude at the point of gelling LFPTEG, in r.u.;

A4 - amplitude LFPTEG after 10 minutes reaching attaining point of gelling, in r.u.;

t5 - time of achievement maximum amplitude LFPTEG (A5) (time of formation a fibrin-platelet clot structure), in minute;

A6 - amplitude LFPTEG after 10 minutes reaching the maximum amplitude, in r.u.

information about the "time slice" state of hemostatic potential (HP) and viability function of the endothelium $[7,13]$.

Picture 2 shows a graph of blood coagulation, obtained by means of TFF, TEG, LFPTEG, TGT, FCMS. From the represented data, of the process of lateral assembly and the formation of cross-linked fibrin takes $\approx 30 \pm 5$ minutes and is determined by almost all methods. "Point of gelling" (blood coagulation time) is the key indicator of the process start of clot formation - transition in the coagulation of blood from a liquid (sol) into a solid (gel). This is estimated only in two ways: FCMS and LFPTEG. Fibrinolytic activity was evaluated by TEG, FCMS and LFPTEG. As for the initial phase of coagulation, its analytical estimation is possible only with the use of LFPTEG. For all other methods, the determined step is regarded as a «lag time» (TGT, TFF), reaction time (TEG), the phase of pre-emerging clot (FCMS) [2].

The graphs (Figure 2) representing the registered blood clotting process to TEG «lag time» until the lateral assembly; for FCMS «lag time» takes place until "gelation point", and the TGT, describing the kinetics of thrombin activity, also has a «lag time» in the time interval 2-3 minutes when setting and within $\approx 30$ minutes due to sample preparation (taking into account the fact that the coagulation process takes place even in the stabilized blood.

Thus, among the "global" tests to evaluate the HP in whole blood, the closest to the observance of the fundamental requirements for the research methods of hemostasis (efficiency, availability, informativity, reliability) is LFPTEG. The results are graphically overlay registration process of whole blood clotting obtained by LFPTEG on a constructed hypothetical model of coagulation. This overlap, reflecting all the stages, was the basis for a new view on system RBA as resulting participation geographically and functionally separates its units (vascular-platelet, coagulation, anticoagulant and fibrinolytic) to ensure proper HP where, in vivo, the process fibrinogenesis in terms of activation, management and restrictions is present permanently (Figure 3).

From positions of system analysis, the resulting functions of the system RBA in vivo is HP of whole blood, and ensuring its consistency over time is determined by nonlinear on time process fibrinogenesis with known and already axiomatic stages of initiation / amplification, propagation, lateral assembly, formation of cross-linked fibrin, until retraction and lysing of the clot. Temporary determinant nonlinearity 
Citation: Solovyev MA, Tutrin II, Udut VV, Borodulina EV, Turenko AV (2015) Global Tests Evaluating the Functional Condition of Hemostasis. Clin Exp Pharmacol 5: 178. doi:10.4172/2161-1459.1000178
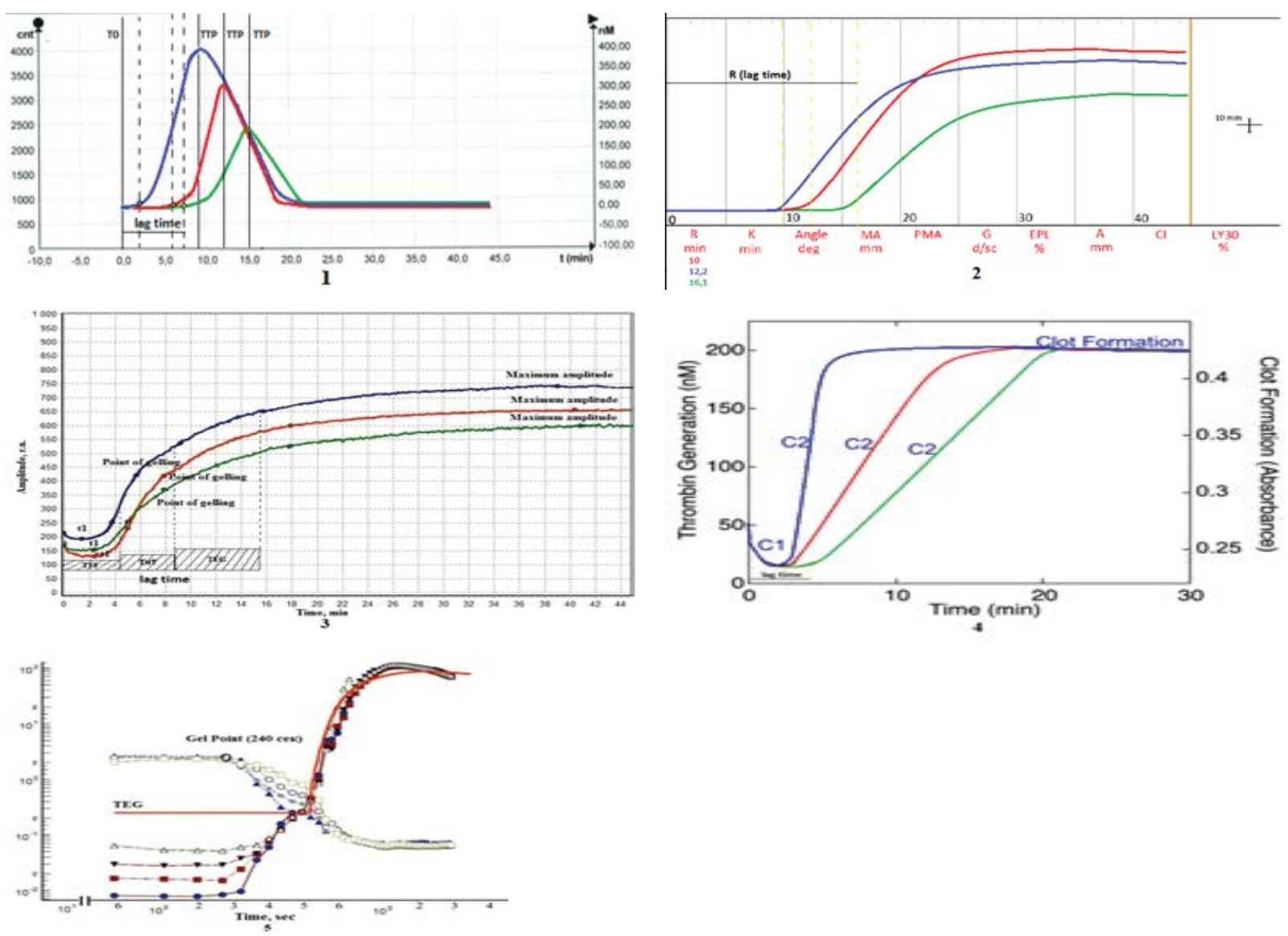

Figure 2: Graphs of blood coagulation, obtained by the methods: 1- THT; 2-TEG; 3- LFNTEG, 4- TFF, 5-FCMS.

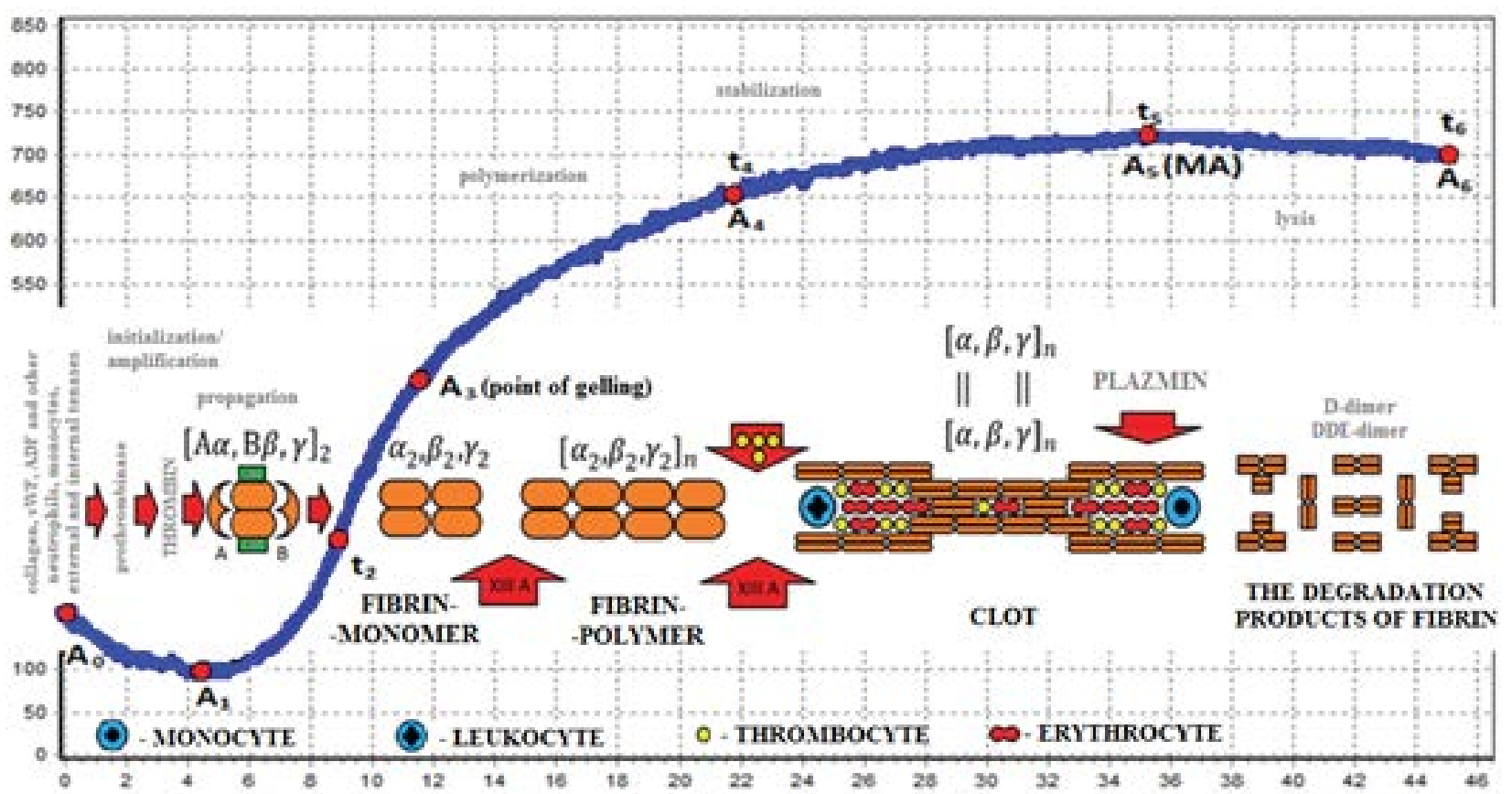

Figure 3: Graph of low-frequency thromboelastography is approximated on a modified version blood coagulation pathway. 
Citation: Solovyev MA, Tutrin II, Udut VV, Borodulina EV, Turenko AV (2015) Global Tests Evaluating the Functional Condition of Hemostasis. Clin Exp Pharmacol 5: 178. doi:10.4172/2161-1459.1000178

fibrinogenesis determined enzymatic potential endothelialintravascular continuum. While not disputing the main provisions of the title role of thrombin in that process it should be understood that it is its amount (level) [14], and most likely - activity [7] that regulated by anticoagulant potential, provides protein $\mathrm{C}$, and $\mathrm{S}$, thrombomodulin, AT III, a2-macroglobulin, NO and prostacyclin, determines the completeness or incompleteness fibrinogenesis in terms of deterrence and limit its rate of formation of metabolic products of fibrinogen and producers of the vascular wall. Thus, in vivo, a second and even a millisecond required for thrombin generation are sufficient to initiate fibrinogenesis, but its viability is directly dependent on the intensity formation and size of thrombin pool, together with the characteristics of blood flow in different parts of vascular channel[19].

\section{Conclusion}

Thus, in view of the existence of a multitude of methods for studying the hemostatic system, it is necessary to distinguish between methods that evaluate the viscoelastic properties of the blood, provided the length of the control scale and timing of deformation, in contrast to other more simple techniques that give the most complete picture of the hemostatic system. Currently, there are a large number of devices that allow for LFPTEG, TEG, TGT, etc., but we must remember that it is necessary to strive for maximum production of early research results system of RBA, close to the bedside, and receive all the information about the picture forming pre-emerging bunch.

\section{References}

1. Dolgov VV, Svirin PV (2005) Laboratory diagnosis of hemostatic disorders: monography. LLC Publishing Triad, Moscow, Tver

2. Kiskun AA (2010) Clinical Laboratory: tutorial. M: GEOTAR Media

3. Klimenkova VF, Bochkov YA, Shpisman MN, Tyutrin II (2012) Identification danger of trombosis as an early marker of preeclampsia by low-frequency piezoelectric thromboelastography. Basic Research.

4. . Baluda VP, Baluda MV, Deyanov II, Tlepshukov IK (1995) Physiology of hemostasis. M: Medicine 238.

5. Barkagan ZS, Momot AP, Nyudiamed M (2008) Diagnosis and controlled therapy of hemostasis disorders: monography. 292
6. Tyutrin II, Klimenkova VF, Udut VV (2014) New technology assessment pharmacodynamics antigregation preparations. Experimental and Clinical Pharmacology 77: 21-25

7. Tyutrin II, Udut VV, Klimenkova VF (2013) The hemostatic system in pregnant women with preeclampsia, according to a global low-frequency piezoelectric thromboelastography. Pathogenesis 11: $54-58$

8. Evans PA, Hawkins K, Lawrence M, Williams RL (2008) Rheometry and associated techniques for blood coagulation studies. Medical Engineering and Physics 30: 671-679.

9. Namestnikov UA (2010) Test generation trombombina - integral index of the state of the blood coagulation system. Hematology and Blood Transfusion 55: 32-39.

10. Solovyev MA, Udut VV, Tyutrin II, Karchagina OS (2015) Pharmacodynamics of antiplatelet agents used for correction of thrombotic complications in polytrauma. Medical-biological and social-psychological issues of safety in emergency situations 1: 69-102

11. Hemker HC, Wielders S, Kessels $H$ (1993) Continuous registration of thrombin generation in plasma, its use for the determination of the thrombin potential. Thromb Haemost 70: 617-624.

12. Momot AP (2006) Pathology of hemostasis. The principles and algorithms for clinical and laboratory diagnosis: monography. - SPb.: Form T,. 208

13. Solovyev MA, Udut VV, Tyutrin II, Klimenkova VF (2013) Experience of diagnosis and monitoring of critical hemostasis disorders. Medical-biological and social-psychological issues of safety in emergency situations 4: 55-60

14. Wolberg AS (2007) Trombin generation and fibrin clot structure. Blood Reviews 21: 131-142.

15. Solovyev MA, Udut VV, Tyutrin II (2014) Innovation technology evaluation of effectiveness of antiaggregants, anticoagulants and fibrinolytics. Clinical \& Experimental Cardiology 5: 196.

16. Kairov GT (2011) Diagnosis, treatment and prevention of thrombosis and thromboembolism Tomsk

17. Tyutrin II, Udut VV, Shpisman MN (2013) Low-frequency piezoelectric thromboelastography in diagnosing hemostatic disorders: methodical guidelines for doctors. Siberian State Medical University,Tomsk

18. .Klimenkova VF, Solovyev MA, Ivanova VA (2014) Experience of the diagnosis and monitoring of critical hemostatic disorders. Clinical Anesthesiology \& Intensive Care 2: 53-59.

19. Patent of Russia № 143093. Apparatus for blood analysis: patent on useful model. / Tyutrin II., publ. 10.07.2014 